\title{
CBL0137 administration suppresses human hepatocellular carcinoma cells proliferation and induces apoptosis associated with multiple cell death related proteins
}

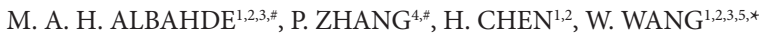 \\ ${ }^{1}$ Department of Hepatobiliary and Pancreatic Surgery, The Second Affiliated Hospital, School of Medicine, Zhejiang University; Hangzhou, \\ China; ${ }^{2}$ Key Laboratory of Precision Diagnosis and Treatment for Hepatobiliary and Pancreatic Tumor of Zhejiang Province, School of Medi- \\ cine, Zhejiang University, Hangzhou, China; ${ }^{3}$ Clinical Research Center of Hepatobiliary and Pancreatic Diseases of Zhejiang Province, School \\ of Medicine, Zhejiang University, Hangzhou, China; ${ }^{4}$ Department of Anesthesiology, Sir Run Run Shaw Hospital, School of Medicine, Zhejiang \\ University, Hangzhou, China; ${ }^{5}$ Clinical Medicine Innovation Center of Precision Diagnosis and Treatment for Hepatobiliary and Pancreatic \\ Disease of Zhejiang University, Hangzhou, China
}

${ }^{*}$ Correspondence: wam@zju.edu.cn

${ }^{*}$ Contributed equally to this work.

Received June 21, 2019 / Accepted September 18, 2019

\begin{abstract}
Hepatocellular carcinoma (HCC) is one of the deadliest cancers worldwide due to the lack of effective therapy methods. Therefore, there is an urgent need to develop novel therapies for HCC. CBL0137 is a small molecule that affects p53 and nuclear factor-kappa B (NF- $\kappa \mathrm{B})$.

The expression of p53 was measured by using immunohistochemistry (IHC) in tumor and adjacent tissues. Western blotting (WB) and quantitative real-time polymerase chain reaction (qRT-PCR) were employed to detect the level of p-p53, p53, Bax, and PUMA after CBL0137 administration. CCK-8 and immunofluorescence staining (IF) assays were performed to evaluate the proliferation and viability of HCC cells. Flow cytometry was used to detect the apoptosis of HCC cells. Xenograft model was established to determine the effect of CBL0137 treatment on HCC tumor growth in vivo. HE staining was used to monitor HCC cell morphology and IHC staining for Ki-67 was performed to determine the tumor cell proliferation following CBL0137 treatment.

Results showed that the expression of p-p53, p53, Bax, and PUMA was upregulated after CBL0137 administration. The viability, growth, and colony formation of HCC cells were significantly inhibited by CBL0137 in the CBL group compared with the NC group $(p<0.05)$. Meanwhile, the results revealed that the proportions of apoptotic and necrotic cells were significantly elevated in the CBL group compared to the NC group $(\mathrm{p}<0.05)$. And the apoptosis-related proteins including PARP, caspase-3, caspase-7, caspase-8, and caspase- 9 were increased in the CBL group compared with the NC group ( $p<0.05)$, while the NF- $\kappa B, p-N F-\kappa B$ and $p-A K T$ expression levels were significantly downregulated following CBL0137 treatment $(\mathrm{p}<0.05)$. Additionally, the tumor volume and weight were significantly reduced in the CBL group compared with the NC group $(\mathrm{p}<0.05)$. Moreover, HE staining and IHC staining for Ki-67 indicated that CBL0137 treatment could obviously induce cell apoptosis and suppress cell proliferation. CBL0137 treatment could effectively inhibit HCC cell proliferation and induce cell apoptosis associated with multiple factors expression.
\end{abstract}

Key words: CBL0137, human hepatocellular carcinoma, proliferation, apoptosis

Hepatocellular carcinoma (HCC) is one of the most prevalent malignancies and the second most lethal cancer worldwide [1]. Approximately 782,500 new cases were diagnosed in 2012, and 746,000 liver cancer-related deaths accounted for $9.1 \%$ of all cancer deaths globally [2]. Researches showed that hepatitis virus infection, alcohol abuse, aflatoxin contamination, and gene susceptibility were the principal causes of HCC [3]. Generally, hepatectomy, liver transplantation, biotherapy, radiotherapy, and chemotherapy are the mainstay of treating HCC for decades [4]. However, the morbidity and mortality of HCC still remain high derived from recurrence and metastasis of the disease. Thus, there is an urgent need for developing new anticancer drugs that effectively treat HCC [5]. Recent studies suggested that 
targeting certain pivotal molecules could reactivate or inhibit several signaling pathways and thereby induce the apoptosis of HCC cells [6].

p53, the first identified tumor suppressor, responds to various critical cellular processes [7]. p53 activation is required to maintain the molecular and genome stability by regulating the expression of target genes, including DNA repair, apoptotic changes, cell cycle, metabolism regulation, and cellular stress $[8,9]$. p53 is usually downregulated in normal cells, while p53 is activated under physical or chemical stress. Inactivation or dysregulation caused by some molecules is one of the most common reasons for inducing HCC [10]. Currently, some researches pointed out that CBL0137 was one of the most promising small molecules and may be useful as a novel treatment for modulating several critical signaling pathways involved in most cancers [11, 12]. CBL0137 has attracted much attention as antitumor drug candidate due to it can simultaneously activate p53 by regulating the FACT (facilitates chromatin transcription) factor and suppressing nuclear factor $(\mathrm{NF}-\kappa \mathrm{B})$ without inducing genotoxicity $[13,14]$. CBL0137 exerts antitumor effects by indirectly inhibiting FACT banded to DNA. As a result, FACT production increases, and thereby results in tumor cell death. CBL0137 exerts crucial anticancer activity in mice with lymphoma without causing systemic toxicity or genotoxicity and also prevents or delays mammary tumors. Moreover, recent studies have shown that CBL0137 can effectively treat colorectal adenocarcinoma, renal cell carcinoma, melanoma, small-cell lung cancer, and pancreatic ductal adenocarcinoma in animal models [15-17]. Thus, we speculated that CBL0137 might be served as a novel agent for the treatment of HCC. In the present study, we attempted to investigate the effect of CBL0137 administration on HCC. The results suggested that CBL0137 could obviously suppress HCC cell proliferation and induce apoptosis by regulating multiple apoptosis-related proteins. Thus, these findings provide a basis for the development of more effective strategies for treating HCC.

\section{Materials and methods}

Patients and tissue samples. Fifty patients with HCC were selected, and cancer and paracancerous tissues were obtained at The First Affiliated Hospital of Zhejiang University (Hangzhou, China) from January 2014 to October 2016. The diagnosis of HCC was confirmed through clinical evidence and pathological diagnosis. These patients had not been treated with chemotherapy and radiotherapy prior to the study and written informed consent was obtained from all voluntary subjects. The participant cohort was 56-78 years old (mean age: $64.36 \pm 18.24$ years). This study was approved by the Human Research Ethics Committee of The First Affiliated Hospital, College of Medicine, Zhejiang University (Hangzhou, China). A total of 50 cancerous and paracancerous sections were collected from each patient with
HCC. Then, formalin-fixed and paraffin-embedded specimens were used for immunohistochemical (IHC) analysis.

Cell lines. The HCC cell lines (including HCCLM3, SMMC-7721, and Huh7) were purchased from the Cell Bank of the Chinese Academy of Sciences (Shanghai, China). The cells were cultured in Dulbecco's modified Eagle's medium (Thermo Fisher Scientific, Waltham, MA, USA) with $10 \%$ fetal bovine serum (Sigma-Aldrich, St. Louis, MO, USA) and incubated in the $37^{\circ} \mathrm{C}$ constant temperature incubator containing $5 \% \mathrm{CO}_{2}$. Subsequent experiments were performed when the cells were at the logarithmic growth stage.

Immunohistochemistry (IHC) staining. Paraffinembedded tissue sections of $5 \mu \mathrm{m}$ thickness were deparaffinized and rehydrated, then antigen retrieval was conducted in $10 \mathrm{mmol} / \mathrm{l}$ citric acid buffer at $100^{\circ} \mathrm{C}$ for $15 \mathrm{~min}$. After incubation with an anti-p53 antibody (\#2527, 1:200; Cell Signaling Technology (Danvers, MA, USA) at $4{ }^{\circ} \mathrm{C}$ overnight, the sections were rinsed with phosphate-buffered saline (PBS) and incubated with a goat anti-rabbit IgG secondary antibody (ab6112, 1:500; Abcam, Cambridge, MA, USA) at $37^{\circ} \mathrm{C}$ for $30 \mathrm{~min}$. The slides were rinsed with PBS, incubated with 3,3'-diaminobenzidine for $2 \mathrm{~min}$, rinsed, and stained with hematoxylin. p53 expression was evaluated by three observers in a blind manner. Five fields were randomly selected and scored for each section. Staining intensity was quantified as follows: no staining $=0$, weak staining $=1$, moderate staining $=2$, and strong staining $=3$; the extent of staining was defined as follows: $0 \%=0,1-24 \%=1,25-49 \%=$ $2,50-74 \%=3$, and $75-100 \%=4$. The final immune-reactive score $(0-12)$ was determined by multiplying the intensity score by the extent of the staining score.

For xenograft IHC analysis, the tumor specimens were fixed in $10 \%$ buffered formalin for $24 \mathrm{~h}$ proceeding to being transferred to $70 \%$ ethanol. Paired paraffin-embedded tissues were used for immunohistochemical staining. A tissue block was cut into $5 \mu \mathrm{m}$ sections, deparaffinized, and rehydrated. Antigen retrieval was conducted in $10 \mathrm{mmol} / \mathrm{l}$ citric acid buffer at $100^{\circ} \mathrm{C}$ for $15 \mathrm{~min}$. After incubation with a primary Ki-67 antibody (ab15580, 1:500; Abcam, Cambridge, MA, USA) at $4{ }^{\circ} \mathrm{C}$ overnight, the sections were rinsed with phosphate-buffered saline (PBS) and incubated with antirabbit IgG secondary antibody (ab6112, 1:500; Abcam, Cambridge, MA, USA) at $37^{\circ} \mathrm{C}$ for $30 \mathrm{~min}$. The slides were washed with PBS, incubated with 3,3'-diaminobenzidine for $2 \mathrm{~min}$, rinsed, and stained with hematoxylin.

Western blotting. After treating with CBL0137, HCC cell lines (including HCCLM3, SMMC-7721, and Huh7) were homogenized with RIPA buffer (Thermo Fisher Scientific, Waltham, MA, USA) containing $1 \%$ protease inhibitor cocktail (Selleck Chemicals, Houston, TX, USA) and phosphatase inhibitors (Sigma-Aldrich, St. Louis, MO, USA) at $4^{\circ} \mathrm{C}$. After centrifugation $\left(14,000 \times \mathrm{g}, 4^{\circ} \mathrm{C}\right)$ for $15 \mathrm{~min}$, the protein concentrations were detected using a bicinchoninic acid assay (Pierce, Rockford, IL, USA) according to the manufacturer's instructions. Equal quantities of proteins were loaded 
and separated by electrophoresis in $12 \%$ sodium dodecyl sulfate polyacrylamide gels (Invitrogen, Carlsbad, CA, USA), and transferred to a polyvinylidene difluoride membrane (PVDF; Millipore, Bedford, MA, USA). The membrane was blocked with $5 \%$ non-fat milk in Tris-buffered saline containing Tween 20 and then incubated at $4{ }^{\circ} \mathrm{C}$ overnight with primary antibodies. The primary antibodies used in this experiment consisting of anti-phospho-p53 (p-p53, \#2521, 1:1000), anti-p53 (\#2527, 1:1000), anti-protein kinase B (also known as AKT, \#4685, 1:1000), anti-phosphorylated AKT (p-AKT, Ser473, \#4060, 1:1000) anti-phospho-NF-kB (p-NF- $\kappa B, \# 3039,1: 1000)$, anti-NF- $\kappa B$ (\#8242, 1:1000, Cell Signaling Technology Danvers, MA, USA); apoptosis-related proteins (including poly(ADP-ribose) polymerase (PARP) (ab74290) at 1:1000, cleaved-PARP (ab32064, 1:1000), caspase-3 (ab32150, 1:1000), caspase-7 (ab32522, 1:1000), caspase-8 (ab32397, at 1:1000), and caspase-9 (ab32539, 1:1000, Abcam, Cambridge, MA, USA). and anti-glyceraldehyde 3-phosphate dehydrogenase (GAPDH, ab8245, 1:1000, Abcam, Cambridge, MA, USA). Therefore, the membranes were further incubated with secondary antibodies for $1 \mathrm{~h}$ at room temperature. Immunodetection was visualized with an EZ-ECL chemiluminescence detection kit (Biological Industries, Beit Haemek, Israel). Protein expression was analyzed using ImageJ software, and GAPDH served as an internal control.

Quantitative real-time polymerase chain reaction (qRT-PCR). The mRNA of Bax and PUMA were detected by using qRT-PCR. Briefly, total RNA was extracted from HCCLM3 cells after CBL0137 administration using TRIzol reagent (superfecTRITM) according to the manufacturer's instructions (Invitrogen), and reverse transcribed to cDNA with the Revert Aid TM First Strand cDNA Synthesis kit (Thermo Fisher Scientific, Waltham, MA, USA). The expression levels were detected using SYBR Premix Ex Taq II kit (Takara Biotechnology Co. Ltd., Dalian, China), and each reaction system contained $2 \mu \mathrm{cDNA}$ template, $0.8 \mu \mathrm{l}$ of each forward and reverse primers $(10 \mathrm{nM}), 6 \mu \mathrm{l}$ RNase-free water, $0.4 \mu \mathrm{l}$ ROX II, and $10 \mu \mathrm{l}$ SYBR Green premix Ex Taq II (Tli RNaseH Plus) in a final volume of $20 \mu \mathrm{l}$. The primers used in the study were as follows: PUMA forward 5'-GCAGCACTTAGAGTCGCC- 3 ' and reverse $5{ }^{`}$-CCTGGGTAAGGGGAGGAGT-3'; Bax forward $5{ }^{\prime}$-CCCGAGAGGTCTTTTTCCGAG-3' and reverse 5'-CCAGCCCATGATGGTTCTGAT-3`; GAPDH forward, $5{ }^{\prime}$-GGAGCGAGATCCCTCCAAAAT-3' and reverse $5{ }^{\prime}$-GGCTGTTGTCATACTTCTCATGG-3`. GAPDH served as a reference gene.

Cell viability assay. HCC cell viability was measured using the Cell Counting Kit (CCK)-8 (Dojindo, Kumamoto, Japan). HCC cells were seeded into 96-well plates and cultured at $37^{\circ} \mathrm{C}$ overnight. The medium was replaced by a complete medium appending with different concentrations of CBL0137, then incubated for an additional $48 \mathrm{~h}$. Next, $10 \mu \mathrm{l}$ CCK-8 solution was added, and the plates were incubated for another $1 \mathrm{~h}$. The absorbance of the colored solutions was quantified using an ELx800 microplate reader (BioTek, Winooski, VT, USA) at $450 \mathrm{~nm}$. Therefore, half-maximal inhibitory concentration $\left(\mathrm{IC}_{50}\right)$ was determined using the GraphPad Prism 5.0 software (GraphPad Software, Inc., La Jolla, CA, USA). Subsequently, HCC cells were treated with CBL0137 at the $\mathrm{IC}_{50}$ value, and the CCK-8 assay was performed to measure the cell proliferation at $24 \mathrm{~h}, 48 \mathrm{~h}, 72 \mathrm{~h}$, and $96 \mathrm{~h}$. Cell proliferation was quantified by measuring OD values at $450 \mathrm{~nm}$, and the proliferation curves were plotted.

Colony formation assay. The HCC cell lines (including HCCLM3, SMMC-7721, and Huh7) were seeded into 6-well plates $\left(2 \times 10^{3}\right.$ cells/well $)$ and cultured at $37^{\circ} \mathrm{C}$ overnight. The medium was replaced by a complete medium containing CBL0137 at the $\mathrm{IC}_{50}$ concentration. The HCC cells were cultured until clones could be observed in naked eyes. The cells were washed twice times with PBS and fixed with methanol after staining with $1 \%$ crystal violet.

Immunofluorescence staining. Briefly, HCCLM3 cells were cultured overnight to attach the glass slides. Then, the cells grown on coverslips were fixed in $4 \%$ paraformaldehyde for $10 \mathrm{~min}$ and permeabilized with $0.3 \%$ Triton X-100 for $15 \mathrm{~min}$ at room temperature. After $30 \mathrm{~min}$ of blocking with $3 \%$ bovine serum albumin (BSA), the cells were incubated with a Ki-67 antibody (Abcam, Cambridge, MA, USA) overnight at $4{ }^{\circ} \mathrm{C}$ and stained with secondary antibodies following day. Nuclei were counterstained with DAPI prior to imaging with the fluorescence microscopy (Olympus, IX71). The number of Ki-67-positive cells was semi-quantified by ImageJ software.

Flow cytometry. Cell apoptosis was detected via using an Annexin V-fluorescein isothiocyanate (FITC) apoptosis detection kit (JingMei Biotech Co., Beijing, China) according to the manufacturer's instructions. HCC cells were seeded into 6-well plates and cultured at $37^{\circ} \mathrm{C}$ overnight to allow adherence. Next, the medium was replaced by a complete medium including CBL0137 at the $\mathrm{IC}_{50}$ concentration, and the cells were then cultured for additional $48 \mathrm{~h}$, followed by treatment with trypsin and incubation with Annexin $\mathrm{V}$ and propidium iodide (PI) at room temperature for 15 min according to the Annexin-V FITC Apoptosis Detection Kit (JingMei Biotech, Beijing, China) following the manufacturer's protocol. Cell apoptosis was evaluated by flow cytometry (FACScan Beckman Instruments, Fullerton, CA, USA). Both Annexin V-FITC and PI were excited at $488 \mathrm{~nm}$, and Annexin V-FITC was detected at $530 \mathrm{~nm}$, and PI was detected at $620 \mathrm{~nm}$. Data were analyzed using the FlowJo software (version 10.0; FlowJo, LLC). Flow cytometry diagrams revealed that living cells (Annexin V-/PI-) were in the lower left quadrant, necrotic cells (Annexin V-/PI+) were in the upper left quadrant, apoptotic cells were in the upper right quadrant (late apoptotic cells: Annexin $\mathrm{V}+/ \mathrm{PI}+$ ) and lower right quadrant (early apoptotic cells: Annexin V+).

Xenograft tumor growth studies. For xenografts, female $\mathrm{BALB} / \mathrm{c}$ nude mice (4-5 weeks old) were purchased from 
SLAC Laboratory Animal Co., Ltd. (Shanghai, China). All animals were housed under a specific pathogen-free facility and maintained based on the guidelines for laboratory animal care. All study protocols were approved by the Zhejiang Medical Experimental Animal Care Commission. Briefly, a suspension of HCCLM3 cells $\left(5 \times 10^{6}\right.$ cells $)$ in $0.2 \mathrm{ml}$ PBS was injected subcutaneously into the right flanks of the mice. Once the tumor volume had reached about $200 \mathrm{~mm}^{3}$, the mice were randomly divided into the untreated control group and CBL0137 group $(\mathrm{N}=6)$. After diluted in mineral oil, an equal dose of DMSO or CBL0137 (10 mg/kg) was injected intraperitoneally into mice every two days (1, 3, and 5 days) for three times. The mice were monitored once every three days. Then mice were sacrificed 3 weeks later, and the related data (weight, length, and width of the tumor) were recorded. The tumor volume was calculated using the following formula: volume $=\left(\mathrm{L} \times \mathrm{W}^{2}\right) / 2$, where $\mathrm{L}$ and $\mathrm{W}$ were the longest and shortest diameters of the tumor, respectively.

HE staining. For histologic analysis, xenograft tumor specimen injected with HCCLM3 cells was fixed into 4\% paraformaldehyde, embedded in paraffin, and cut into $5 \mu \mathrm{m}$ thick slides. The slides were dyed by hematoxylin and eosin (H\&E) for histomorphometric analyses. Images were captured by a microscope (Olympus BX51).

Statistical analysis. All statistical analyses were performed by using the SPSS 17.0 software (SPSS, Chicago, IL). And the data were presented as the mean \pm standard deviation (SD). A p-value $<0.05$ was considered statistically significant, and all experiments were carried out at least in triplicates.

\section{Results}

Multiple factors were reactivated after the CBL0137 administration. The expression of p53 was measured by using IHC staining in 50 cases of available matched HCC and adjacent tissue specimens. The results showed that $\mathrm{p} 53$ staining was mainly located in the cell nucleus, and the level of p53 in tumor tissues was significantly higher than that of in peritumoral samples (Figure 1A). Meanwhile, qRT-PCR and $\mathrm{WB}$ were separately employed to investigate whether the CBL0137 administration could reactivate extra molecules expression. The results showed that the protein expression of p-p53 and total p53 was significantly upregulated following treating with $\mathrm{CBL} 0137$ in the $\mathrm{CBL}$ group (including HCCLM3, SMMC-7721, and Huh7) than that of in the NC group (Figures $1 \mathrm{~B}-\mathrm{C}, \mathrm{p}<0.05$ ). And the mRNA expression of Bax and PUMA was higher in the CBL group (HCCLM3 cells) when compared with the NC group (Figures 1D-E, $\mathrm{p}<0.05)$.

CBL0137 administration inhibited HCC cell proliferation and clonogenic ability in vitro. CCK-8 assay was performed to evaluate the proliferation of HCC cell lines (HCCLM3, SMMC-7721, and Huh7), and $\mathrm{IC}_{50}$ values of CBL0137 were calculated from five replicative experiments of each cell line. As shown in Figure 2A, CBL0137 significantly inhibited the cell growth in a dose-dependent manner, and the $\mathrm{IC}_{50}$ was $0.394 \pm 0.069 \mu \mathrm{M}$ (HCCLM3), $0.298 \pm 0.046 \mu \mathrm{M}$ (SMMC-7721), and $0.383 \pm 0.053 \mu \mathrm{M}$ (Huh7), respectively. Meanwhile, the cell proliferation was obviously suppressed in the CBL group (HCCLM3, SMMC-7721, and Huh7) at $24 \mathrm{~h}$, $48 \mathrm{~h}, 72 \mathrm{~h}$, and $96 \mathrm{~h}$ after the CBL administration compared with the NC group (Figures $2 \mathrm{~B}-\mathrm{D}, \mathrm{p}<0.05$ ). Moreover, the $\mathrm{CBL}$ treatment significantly suppressed the colony formation in the CBL group (HCCLM3, SMMC-7721, and Huh7) compared to the NC group (Figures $2 \mathrm{E}-\mathrm{F}, \mathrm{p}<0.05)$. The expression of proliferation index Ki-67 was detected by immunofluorescence assay, and the results suggested that the level of Ki-67 was reduced in the CBL group (HCCLM3 cells) compared with the NC group (Figures $2 \mathrm{G}-\mathrm{H}, \mathrm{p}<0.05$ ).

CBL0137 administration induces apoptosis of HCC cells in vitro. HCC cells (HCCLM3, SMMC-7721, and Huh7) were treated using CBL0137 with $\mathrm{IC}_{50}$ concentration for $24 \mathrm{~h}$ to determine the effect of CBL0137 on apoptosis. Annexin V-FITC/PI double-staining apoptosis detection kit and flow cytometry were used to detect the apoptosis degree. As shown in Figure 3A, the left bottom indicated the proportion of normal viable cells, the right bottom showed the proportion of cells in early apoptosis, the upper right exhibited the proportion of cells in late apoptosis and necrocytosis, and the upper left displayed the proportion of cell debris and a few necrotic cells. The results revealed that treatment with CBL0137 increased the proportions of apoptotic cells by 41.05\%, 35.22\%, and 33.94\% in HCCLM3, SMMC-7721, and Huh7 populations, respectively, compared with those in the untreated control groups (Figures $3 \mathrm{~A}-\mathrm{B}, \mathrm{p}<0.05$ ). Additionally, the protein expressions of apoptosis-related proteins including PARP, caspase-3, caspase-7, caspase-8, and caspase- 9 were increased in the $\mathrm{CBL}$ group compared with the NC group (Figure 3C, Figures 3E-G, p<0.05). Meanwhile, the results showed that NF- $\kappa \mathrm{B}, \mathrm{p}-\mathrm{NF}-\kappa \mathrm{B}$, and $\mathrm{p}-\mathrm{AKT}$ expression levels were significantly downregulated following the CBL0137 treatment (Figure 3D, Figures 3H-J, $\mathrm{p}<0.05$ ).

CBL0137 inhibits HCC cell-induced tumor growth in nude mice. A xenograft model in nude mice was established to determine the effect of the CBL0137 administration on HCC tumor growth in vivo. Subcutaneous xenograft tumors were induced by HCCLM3 cell injection in female BALB/c nude mice. The antitumor effect of CBL0137 was measured once every three days. There was no statistical difference in body weight between the CBL group and the NC group (Figure 4A, p>0.05). The tumor volume was significantly reduced in the CBL group compared with the NC group (Figure 4B, p <0.05), and the mean weight of tumor was decreased in the $\mathrm{CBL}$ group compared to the NC group (Figures 4C-D, $\mathrm{p}<0.05$ ). Besides, the CBL0137 treatment obviously elicited cell swelling, chromatin condensation, or nuclear condensation and fragmentation (Figure 4G). In addition, Ki-67 staining was performed to confirm these results (Figures $4 \mathrm{E}-\mathrm{F}, \mathrm{p}<0.05$ ). Collectively, these findings revealed that CBL0137 suppresses HCC progression in vivo. 


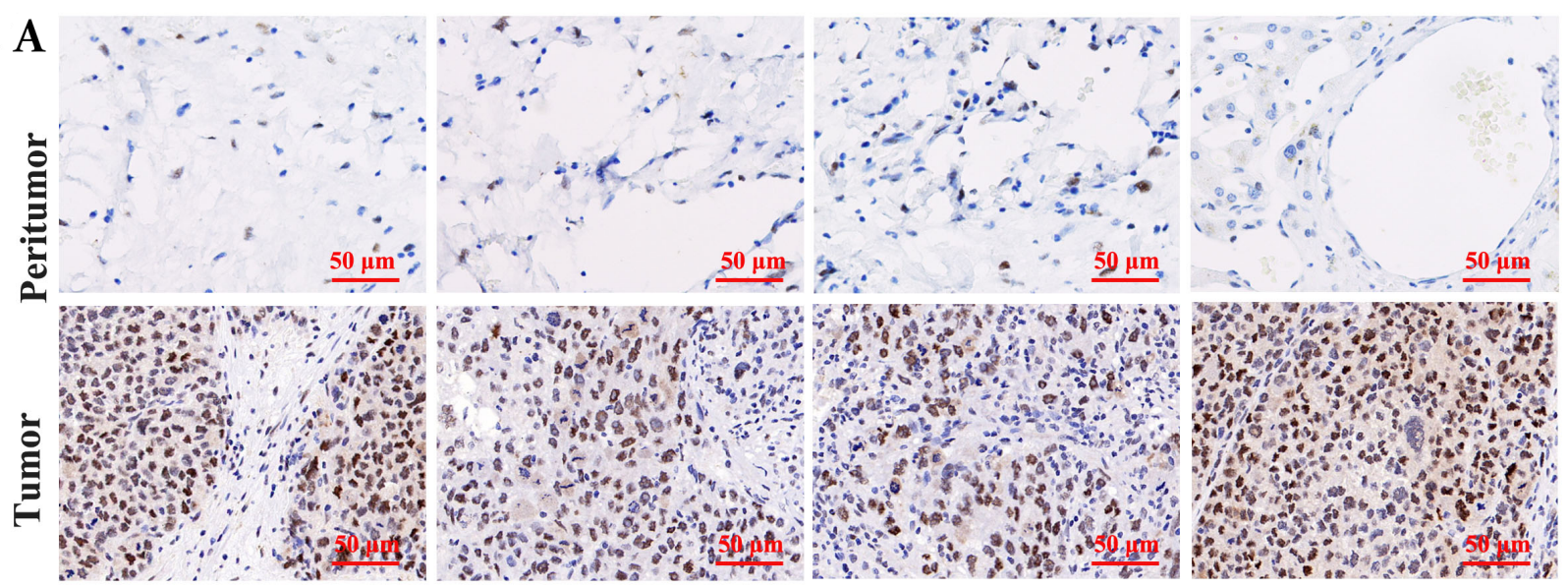

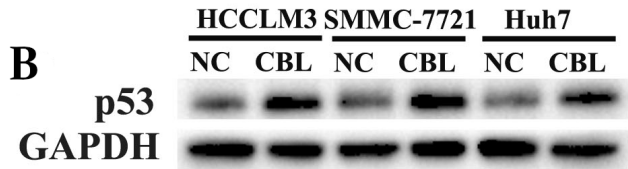

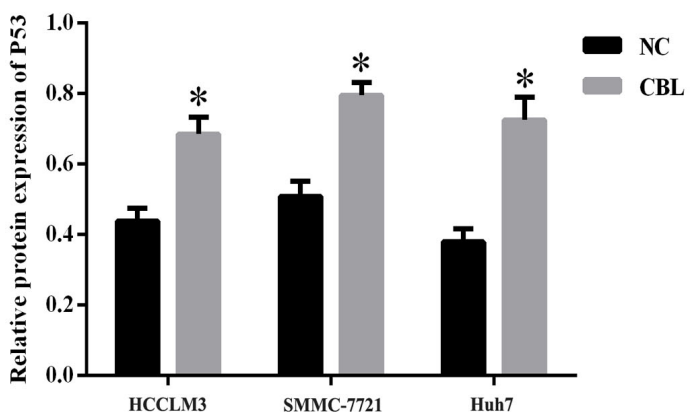

D

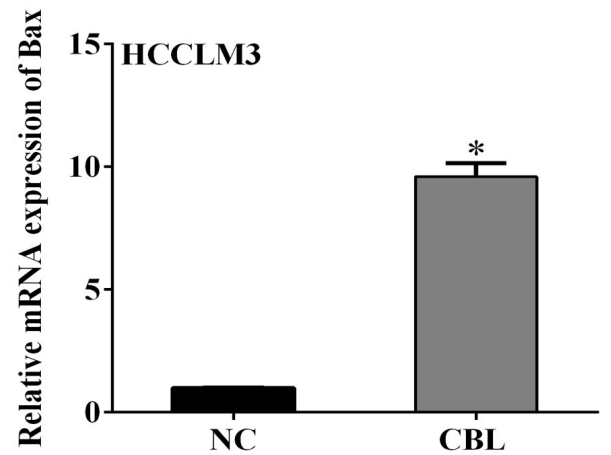

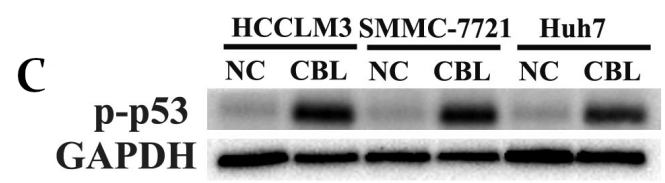
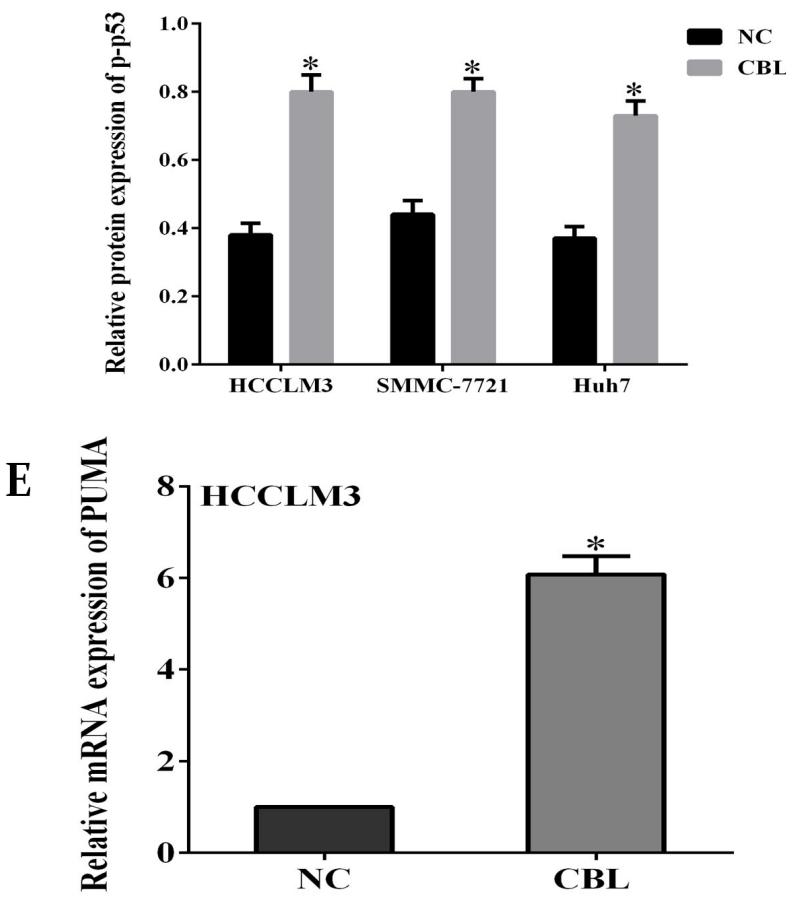

Figure 1. Multiple factors were reactivated after the CBL0137 administration. A) Representative IHC staining for p53 in 4 pairs of HCC tissues and adjacent non-neoplastic liver tissues (magnification $\times 400$ ); B) Western blot analysis for p53 in HCC cell lines including HCCLM3, SMMC-7721, and Huh7 treated with CBL0137. C) Western blot analysis for p-p53 in HCC cell lines including HCCLM3, SMMC-7721, and Huh7 treated with CBL0137. D, E) The mRNA expression of Bax and PUMA. GAPDH was used as an internal control. NC, untreated control; CBL, CBL0137; HCC, hepatocellular carcinoma, Immunohistochemistry, IHC. ${ }^{*} \mathbf{p}<0.05$, compared with NC group.

\section{Discussion}

HCC is a major health problem and one of the most devastating diseases threatening human health all over the world [18]. In the present study, the effects of CBL0137 on HCC progression were evaluated in vitro and in vivo, and the underlying mechanism was further investigated.
Advanced therapeutic strategies for HCC, such as novel molecular targeted therapies, are urgently needed [19]. As we all know that one of the most fundamental characteristics of cancer cells is their ability that maintaining continuous proliferation [20]. Generally, normal tissues could produce and secrete growth-promoting signal molecules to ensure 


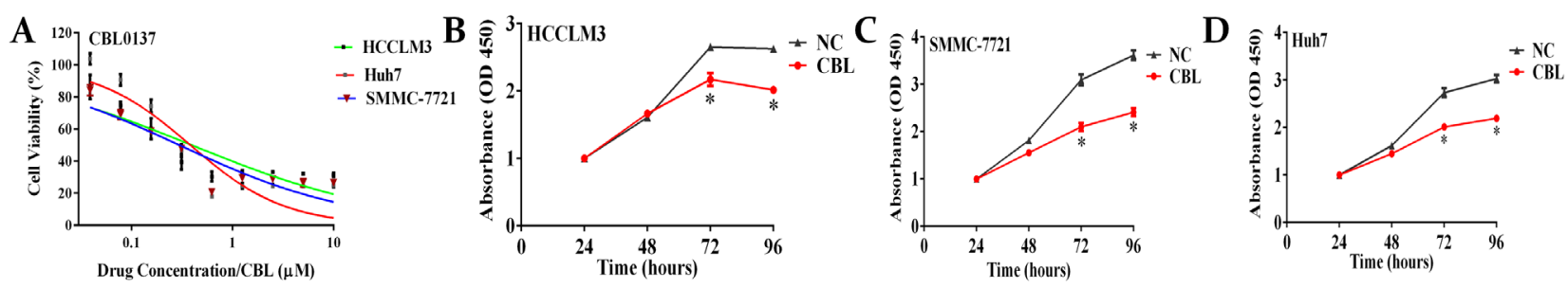

$\mathbf{E}$
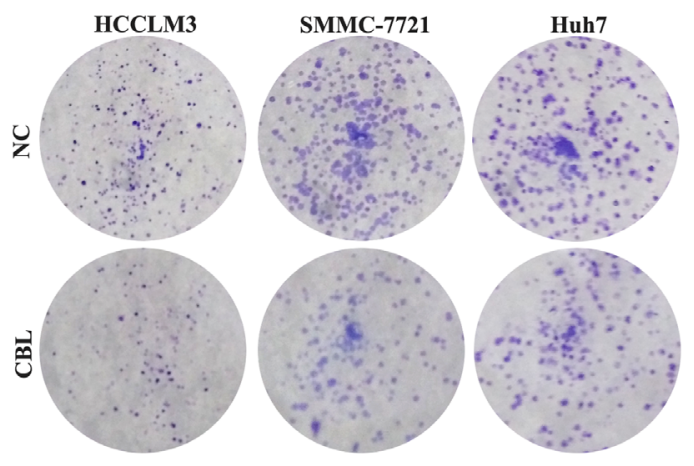

G
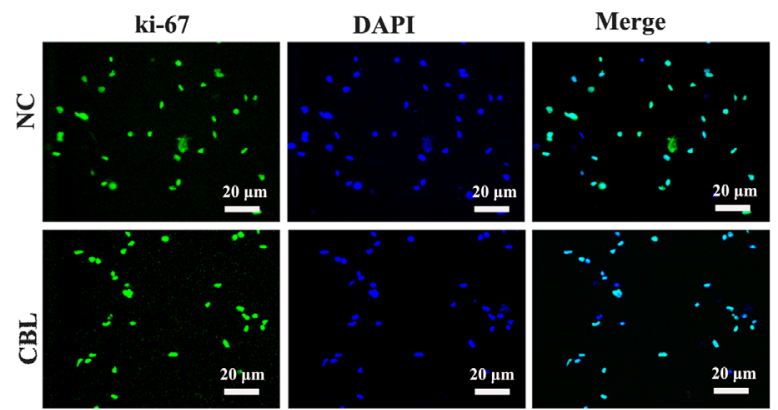

$\mathbf{F}$

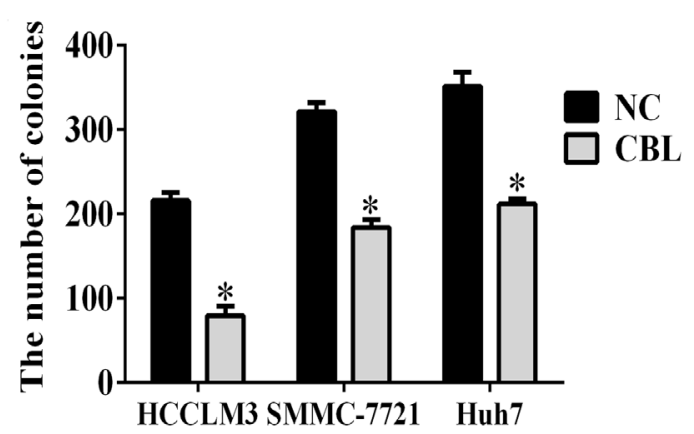

$\mathbf{H}$

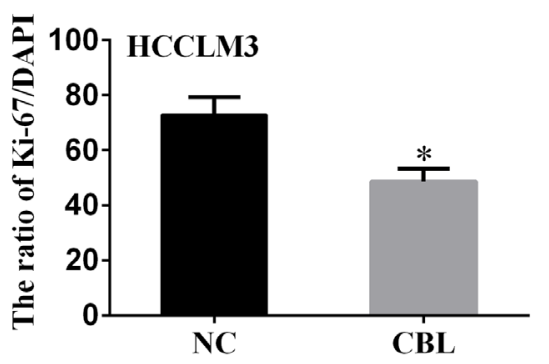

Figure 2. CBL0137 administration inhibited HCC cell proliferation and clonogenic ability in vitro A) Dose-response growth curves of HCC cell lines treated with CBL0137; B-D) Effects of the CBL0137 administration on the HCC cell viability (HCCLM3, SMMC-7721, and Huh7) during 0, 24, 48, 72 hours detected by the CCK-8 assay; E, F) Colony formation abilities of HCC cells (HCCLM3, SMMC-7721, and Huh7); G, H) Expression of Ki-67 on HCCLM3 cells detected by immunofluorescence experiment (magnification $\times 400$ ). NC, untreated control; CBL, CBL0137; HCC, hepatocellular carcinoma. ${ }^{\star} \mathrm{p}<0.05$, compared with $\mathrm{NC}$ group.

homeostasis of the cell number, architecture, and function [21]. However, cancer cells deregulate these signals, which thereby accelerate cell proliferation, adhesion, and invasion. Recent researches found that a multitude of tumor suppressors limit cell growth and proliferation via specific inactivation of intracellular signals in different types of cancers [22]. Some of these molecules have been identified as vital tumor suppressors by functional experiments in mice. p53 is a prototypical tumor suppressor that serves as a central control node determining cell proliferation, senescence or apoptotic programs [23]. High-throughput DNA sequencing analyses had revealed that activation or inactivation of the key genes played a crucial role in the oncogenesis and development of HCC. The dysfunction of key genes would result in tumor cells escape from death and rapid tumor progression $[24,25]$.

CBL0137 is a clinical candidate for a new class of anticancer drugs, which has been tested in clinical trials. Related studies had documented that CBL0137 possessed potent anticancer activities in pancreatic cancer, breast cancer, non-small cell lung cancer, neuroblastoma, colorectal adenocarcinoma, and melanoma $[26,27]$. These results showed that the CBL0137 treatment could significantly suppress HCC cell proliferation and induce apoptosis associated with multiple proteins expression including p53 and its downstream targets. CBL0137 showed an antitumor effect against HCC, and the mechanism was deeply investigated. WB assay showed that apoptosis-related proteins, including caspase-3, caspase-7, caspase-8, caspase-9, and PARP, were upregulated after CBL0137 treatment.

The inactivation of pivotal signaling pathways would lead to loss of its anti-apoptotic function, increase genome instability, and inhibit the unconstrained growth of tumor cells. It has been reported that the expression level of AKT and $\mathrm{NF}-\kappa \mathrm{B}$ is higher in tumor than in non-tumor tissue, which 
A
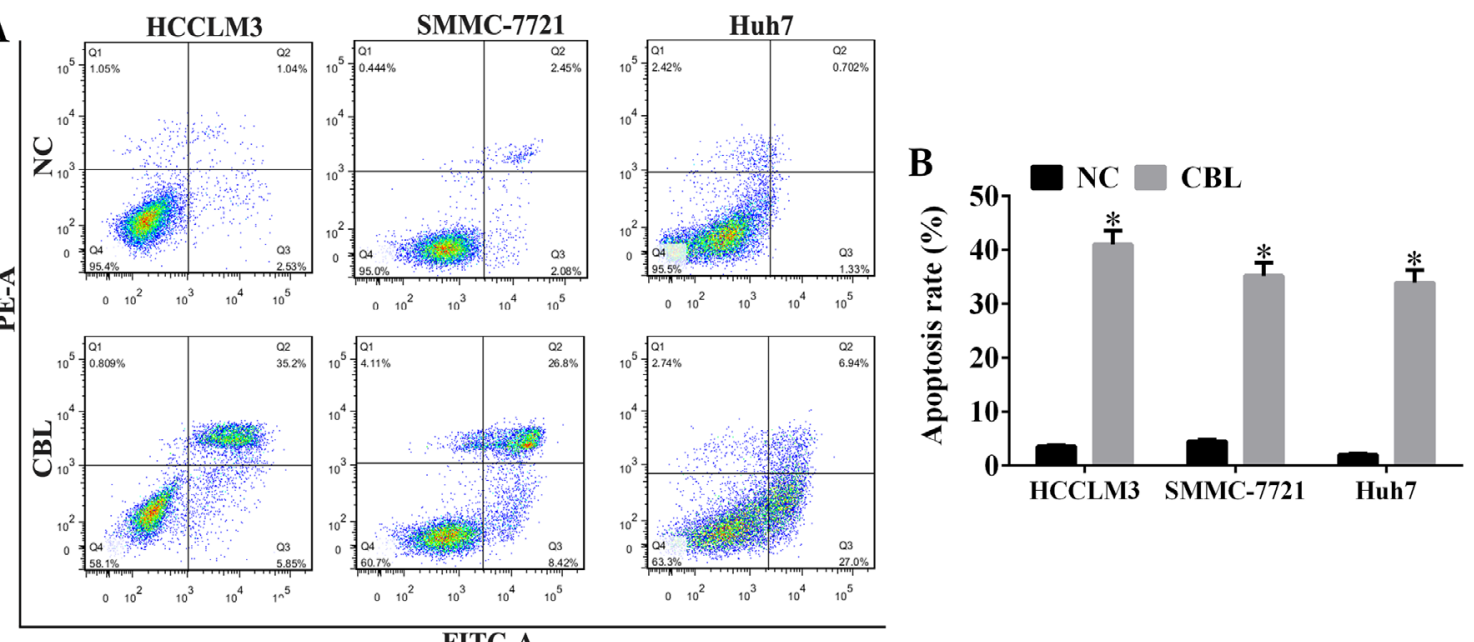

FITC-A

C

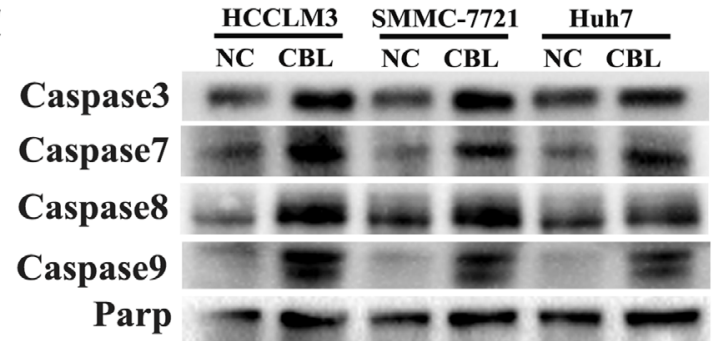

D

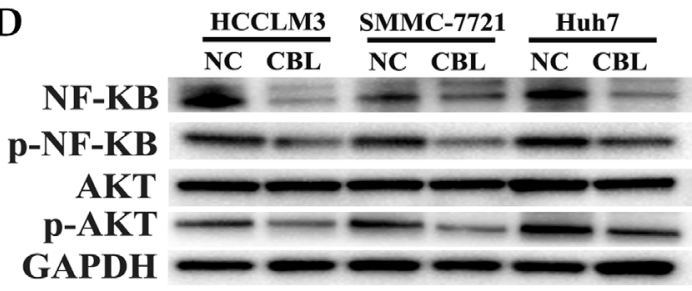

Cleav-Parp

\section{GAPDH}

$\mathbf{E}$

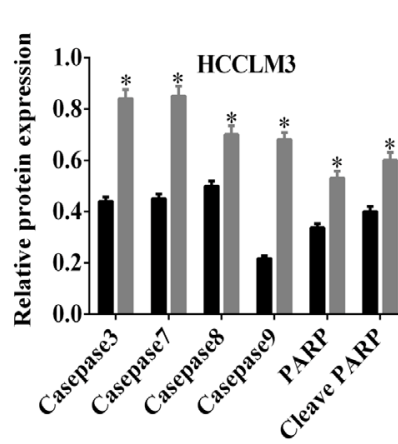

H

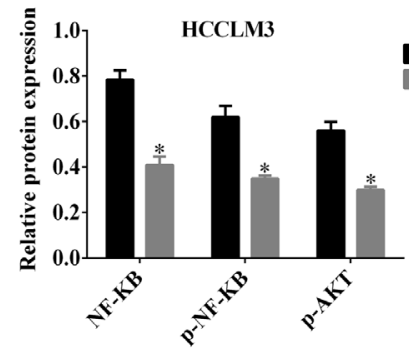

$\mathbf{F}$
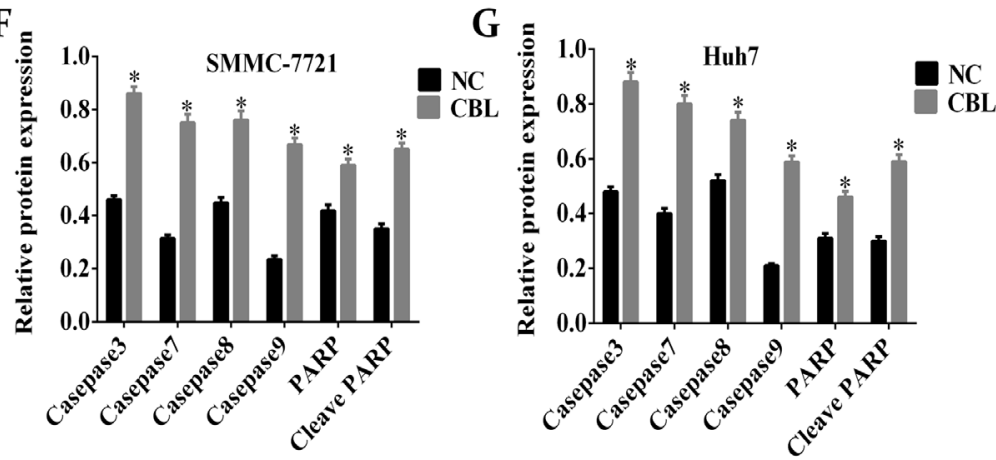

J

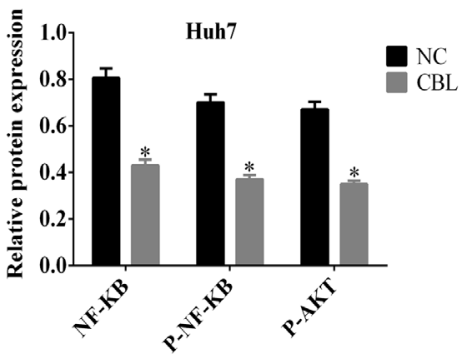

Figure 3. Effect of CBL0137 on the HCC cells. A, B) Flow-cytometric quantification of apoptosis in HCC cells (HCCLM3, SMMC-7721, and Huh7) after treatment with CBL0137; C) Effects of CBL0137 on apoptosis-related protein expression including caspase-3, caspase-7, caspase-8, caspase-9, PARP; D) Effects of CBL0137 on NF-kB, p-NF-кB, AKT, and p-AKT protein expression; E-G) Quantitative results of WB assay concerning apoptosis-related proteins on HCC cells (HCCLM3, SMMC-7721, and Huh7); H-J) Quantitative results of WB assay concerning NF-kB, p-NF- $\mathrm{kB}$, AKT, and p-AKT protein levels on HCC cells (HCCLM3, SMMC-7721, and Huh7) NC, untreated control; CBL, CBL0137; HCC, hepatocellular carcinoma. ${ }^{*}$ p $<0.05$, compared with NC group. 
A
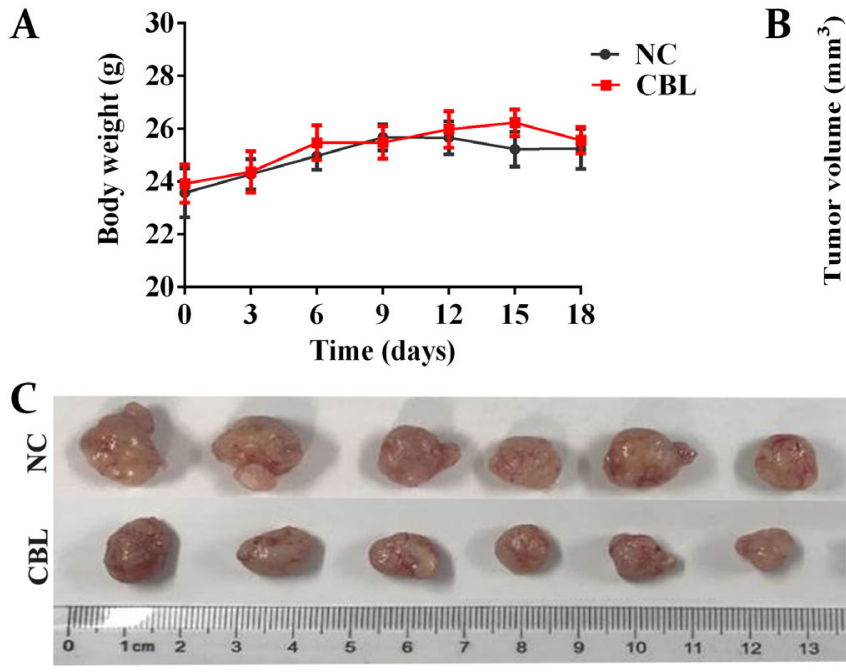

$\mathbf{E}$

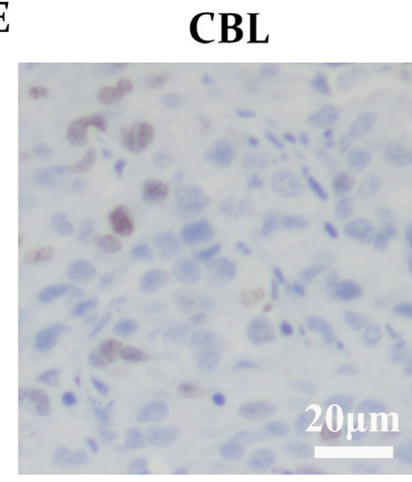

G

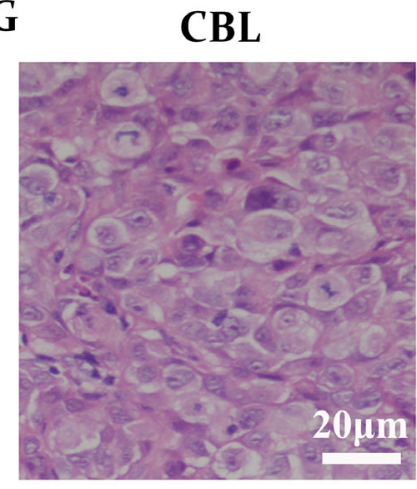

NC

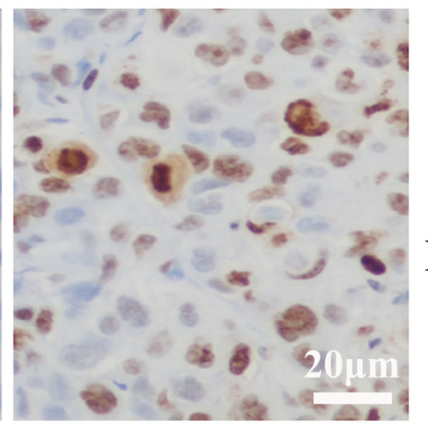

B
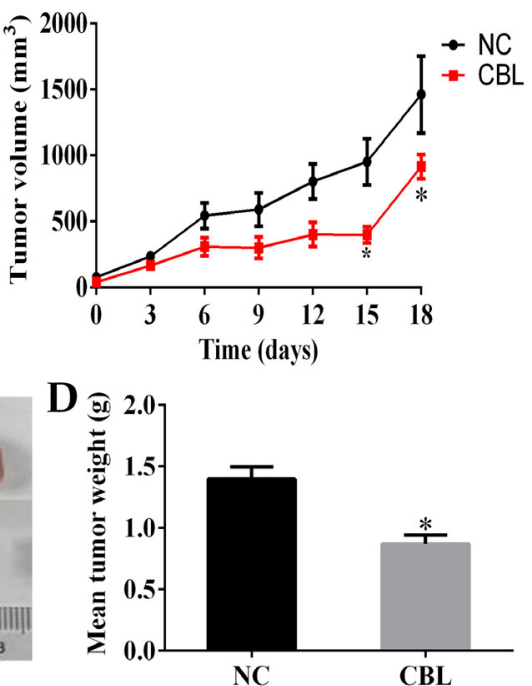

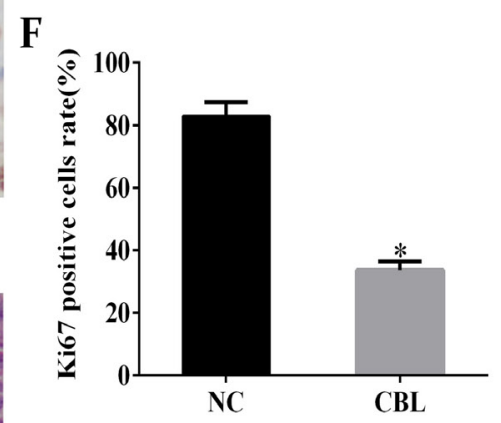

Figure 4. Antitumor efficacy of CBL0137 in HCCLM3 subcutaneous xenograft nude mice. A) Body weight changes after treating with CBL0137; B-D) The effect of CBL0137 on tumor volume and weight; E-F) Representative images for Ki-67 staining and percentage of Ki-67-positive cells in different groups; G) Representative images of HE staining in different groups. NC, untreated control; CBL, CBL0137. ${ }^{*}$ p $<0.05$, compared with NC group, (magnification $\times 400$ ). The Ki-67-positive cells were calculated using ImageJ software.

could regulate cell proliferation and apoptosis [28, 29]. Recent research showed that the NF- $\kappa B$ was constitutively activated in most cancer cells and affected inflammatory and immune responses [30]. Furthermore, NF- $\kappa \mathrm{B}$ is an essential factor affecting the expression of specific cytokines, tumor growth, and chemical/radio-resistance during cancer therapy $[31,32]$. Additionally, numerous studies have demonstrated that AKT is activated and overexpressed in various types of carcinomas such as prostate cancer, breast cancer and ovarian cancer, and shows a positive relationship with tumorigenesis and resistance to chemotherapy $[33,34]$. The present study indicated that the activation of the AKT signaling pathway was significantly correlated with poor prognosis in HCC. Moreover, recent studies confirmed that the AKT pathway 
participated in the regulation of cell proliferation, differentiation, and apoptosis $[35,36]$. Therefore, WB assay was employed to detect the effect of the CBL0137 treatment on the AKT and NF- $\mathrm{KB}$ pathway. Intriguingly, we found that CBL0137 significantly downregulated AKT phosphorylation, NF- $\kappa B$ and $\mathrm{p}-\mathrm{NF}-\kappa \mathrm{B}$ protein expression in HCC cells, which indicated that signaling pathways comprising of AKT and NF- $\kappa B$ may be effectively blocked by CBL0137. Meanwhile, the cell viability was obviously suppressed following the CBL0137 treatment indicated by CCK- 8 assay, and the immunofluorescence assay showed that the expression of proliferation index Ki-67 was reduced after CBL0137 treatment. Moreover, CBL treatment significantly suppressed the colony formation in vitro. Besides, CBL0137 treatment obviously elicited HCC cell apoptosis, suppressed proliferation and ultimately led to the decrease of tumor weight and volume in the HCC xenograft model.

Taken together, the present study revealed that CBL0137 could effectively inhibit cell proliferation and induce apoptosis in HCC. The possible mechanisms related to the activation of apoptosis-related protein and suppress the NF- $\mathrm{\kappa B}$ and AKT pathways. Our findings would eventually lay down a significant theoretic foundation for later academic studies on the anticancer effects of CBL0137 in HCC.

Acknowledgments: This work was supported by the National Natural Science Foundation of China (No. 81572307 and 81773096) and by the Major Project of the Medical and Health Technology Development Program in Zhejiang Province (No. 7211902).

\section{References}

[1] BYAM J, RENZ J, MILLIS JM. Liver transplantation for hepatocellular carcinoma. Hepatobiliary Surg Nutr 2013; 2: 22-30. https://doi.org/10.3978/j.issn.2304-3881.2012.11.03

[2] WALLACE MC, PREEN D, JEFFREY GP, ADAMS LA. The evolving epidemiology of hepatocellular carcinoma: a global perspective. Expert Rev Gastroenterol Hepatol 2015 Jun; 9: 765-779. https://doi.org/10.1586/17474124.2015.1028363

[3] YOSHIMOTO S, LOO TM, ATARASHI K, KANDA H, SATO $S$ et al. Obesity-induced gut microbial metabolite promotes liver cancer through senescence secretome. Nature 2013; 499: 97-101. https://doi.org/10.1038/nature12347

[4] LIM C, SHINKAWA H, HASEGAWA K, BHANGUI P, SALLOUM C et al. Salvage liver transplantation or repeat hepatectomy for recurrent hepatocellular carcinoma: An intent-to-treat analysis. Liver Transpl 2017; 23: 1553-1563. https://doi.org/10.1002/lt.24952

[5] SONG M J, BAE S H. Newer treatments for advanced hepatocellular carcinoma. Korean J Intern Med 2014; 29: 149155. https://doi.org/10.3904/kjim.2014.29.2.149

[6] BHAYANI NH, JIANG Y, HAMED O, KIMCHI ET, STAVELEY-O'CARROLL KF et al. Advances in the Pharmacologic Treatment of Hepatocellular Carcinoma. Curr Clin Pharmacol 2015; 10: 299-304. https://doi.org/10.2174/15748847106 66151020100059
[7] VOUSDEN K H, PRIVES C. Blinded by the Light: The Growing Complexity of p53. Cell 2009; 137: 413-431. https://doi. org/10.1016/j.cell.2009.04.037

[8] KRUSE JP, GU W. Modes of p53 regulation. Cell 2009; 137: 609-622. https://doi.org/10.1016/j.cell.2009.04.050

[9] DEY A, TERGAONKAR V, LANE DP. Double-edged swords as cancer therapeutics: simultaneously targeting p53 and NF-kappaB pathways. Nat Rev Drug Discov 2008; 7: 1031-1040. https://doi.org/10.1038/nrd2759

[10] ARAVALLI RN, STEER CJ, CRESSMAN EN. Molecular mechanisms of hepatocellular carcinoma. Hepatology 2008; 48: 2047-2063. https://doi.org/10.1002/hep.22580

[11] GASPARIAN AV, BURKHART CA, PURMAL AA, BRODSKY L, PAL $M$ et al. Curaxins: anticancer compounds that simultaneously suppress NF- $\mathrm{kB}$ and activate p53 by targeting FACT. Sci Transl Med 2011; 3: 95ra74. https://doi. org/10.1126/scitranslmed.3002530

[12] DI BUSSOLO V, MINUTOLO F. Curaxins: a new family of non-genotoxic multitargeted anticancer agents. ChemMedChem 2011; 6: 2133-2136. https://doi.org/10.1002/ cmdc. 201100476

[13] BURKHART C, FLEYSHMAN D, KOHRN R, COMMANE M, GARRIGAN J et al. Curaxin CBL0137 eradicates drug resistant cancer stem cells and potentiates efficacy of gemcitabine in preclinical models of pancreatic cancer. Oncotarget 2014; 5: 11038-11053. https://doi.org/10.18632/oncotarget.2701

[14] BARONE TA, BURKHART CA, SAFINA A, HADERSKI G, GUROVA KV et al. Anticancer drug candidate CBL0137, which inhibits histone chaperone FACT, is efficacious in preclinical orthotopic models of temozolomide-responsive and -resistant glioblastoma. Neuro Oncol 2017; 19: 186-196. https://doi.org/10.1093/neuonc/now141

[15] DRAETTA GF, DEPINHO RA. Cancer drug discovery faces the FACT. Sci Transl Med 2011; 3: 95ps34. https://doi. org/10.1126/scitranslmed.3002822

[16] GARCIA H, FLEYSHMAN D, KOLESNIKOVA K, SAFINA A, COMMANE $\mathrm{M}$ et al. Expression of FACT in mammalian tissues suggests its role in maintaining of undifferentiated state of cells. Oncotarget 2011; 2: 783-796. https://doi. org/10.18632/oncotarget. 340

[17] KOMAN IE, COMMANE M, PASZKIEWICZ G, HOONJAN B, PAL $S$ et al. Targeting FACT complex suppresses mammary tumorigenesis in Her2/neu transgenic mice. Cancer Prev Res (Phila). 2012; 5: 1025-1035. https:// doi.org/10.1158/1940-6207.CAPR-11-0529

[18] SIEGEL R L, MILLER K D, JEMAL A. Cancer statistics, 2015. CA Cancer J Clin 2015; 65: 5-29. https://doi.org/10.3322/ caac. 21254

[19] GE S, HUANG D. Systemic therapies for hepatocellular carcinoma. Drug Discov Ther 2015; 9: 352-362. https://doi. org/10.5582/ddt.2015.01047

[20] WEBBER J, YEUNG V, CLAYTON A. Extracellular vesicles as modulators of the cancer microenvironment. Semin Cell Dev Biol 2015; 40: 27-34. https://doi.org/10.1016/j. semcdb.2015.01.013

[21] QUAIL D F, JOYCE J A: Microenvironmental regulation of tumor progression and metastasis. Nat Med 2013; 19: 14231437. https://doi.org/10.1038/nm.3394. 
[22] IKEMURA S, ARAMAKI N, FUJII S, KIRITA K, UMEMURA $S$ et al. Changes in the tumor microenvironment during lymphatic metastasis of lung squamous cell carcinoma. Cancer Sci 2017; 108: 136-142. https://doi.org/10.1111/ cas. 13110

[23] GUROVA KV, HILL JE, GUO C, PROKVOLIT A, BURDELYA LG et al. Small molecules that reactivate p53 in renal cell carcinoma reveal a NF-kappaB-dependent mechanism of p53 suppression in tumors. Proc Natl Acad Sci U S A. 2005; 102: 17448-17453. https://doi.org/10.1073/pnas.0508888102

[24] HE X, LIU F, YAN J, ZHANG Y, YAN J et al. Trans-splicing repair of mutant p53 suppresses the growth of hepatocellular carcinoma cells in vitro and in vivo. Sci Rep 2015; 5: 8705. https://doi.org/10.1038/srep08705

[25] LI Q, LIU X, JIN K, LU M, ZHANG C et al. NAT10 is upregulated in hepatocellular carcinoma and enhances mutant p53 activity. BMC Cancer 2017; 17: 605. https://doi.org/10.1186/ s12885-017-3570-4

[26] DE S, LINDNER DJ, COLEMAN CJ, WILDEY G, DOWLATI A et al. The FACT inhibitor CBL0137 Synergizes with Cisplatin in Small-Cell Lung Cancer by Increasing NOTCH1 Expression and Targeting Tumor-Initiating Cells. Cancer Res 2018; 78: 2396-2406. https://doi.org/10.1158/0008-5472. CAN-17-1920

[27] FLEYSHMAN D, PRENDERGAST L, SAFINA A, PASZKIEWICZ G, COMMANE $M$ et al. Level of FACT defines the transcriptional landscape and aggressive phenotype of breast cancer cells. Oncotarget 2017; 8: 20525-20542. https:// doi.org/10.18632/oncotarget.15656

[28] LI W, TAN D, ZENALI MJ, BROWN RE. Constitutive activation of nuclear factor-kappa B (NF-kB) signaling pathway in fibrolamellar hepatocellular carcinoma. Int J Clin Exp Pathol 2009; 3: 238-243.

[29] WARDLE EN. Nuclear factor kappaB for the nephrologist. Nephrol Dial Transplant 2001; 16: 1764-1768. https://doi. org/10.1093/ndt/16.9.1764
[30] LIU YC, CHIANG IT, HSU FT, HWANG JJ. Using NF-kap$\mathrm{paB}$ as a molecular target for theranostics in radiation oncology research. Expert Rev Mol Diagn 2012; 12: 139-146. https://doi.org/10.1586/erm.12.2

[31] KUO YC, LIN WC, CHIANG IT, CHANG YF, CHEN CW et al. Sorafenib sensitizes human colorectal carcinoma to radiation via suppression of NF-kappaB expression in vitro and in vivo. Biomed Pharmacother 2012; 66: 12-20. https://doi. org/10.1016/j.biopha.2011.09.011

[32] CHIANG IT, LIU YC, HSU FT, CHIEN YC, KAO CH et al. Curcumin synergistically enhances the radiosensitivity of human oral squamous cell carcinoma via suppression of radiation-induced NF-kappaB activity. Oncol Rep 2014; 31: 1729-1737. https://doi.org/10.3892/or.2014.3009

[33] POLIVKA J JR, JANKU F. Molecular targets for cancer therapy in the PI3K/AKT/mTOR pathway. Pharmacol Ther 2014; 142: 164-175. https://doi.org/10.1016/j.pharmthera.2013.12.004

[34] SCHMITZ KJ, WOHLSCHLAEGER J, LANG H, SOTIROPOULOS GC, MALAGO M et al. Activation of the ERK and AKT signalling pathway predicts poor prognosis in hepatocellular carcinoma and ERK activation in cancer tissue is associated with hepatitis C virus infection. J Hepatol 2008; 48: 83-90. https://doi.org/10.1016/j.jhep.2007.08.018

[35] LU Q, YAN S, SUN H, WANG W, LI Y et al. Akt inhibition attenuates rasfonin-induced autophagy and apoptosis through the glycolytic pathway in renal cancer cells. Cell Death Dis 2015; 6: e2005. https://doi.org/10.1038/cddis.2015.344

[36] WANG Z, ZHOU L, ZHENG X, CHEN G, PAN R et al. Autophagy protects against PI3K/Akt/mTOR-mediated apoptosis of spinal cord neurons after mechanical injury. Neurosci Lett 2017; 656: 158-164. https://doi.org/10.1016/j. neulet.2017.07.036 